\title{
A PRÁTICA DA AMAMENTAÇÃO NO MUNICtPIO DE PAULINIA, ESTADO DE SÃO PAULO, BRASIL
}

\author{
Ellen E. Hardy * \\ Regina Sarmento * \\ Martha Gushiken * \\ Ronaldo Araki ** \\ José Martins Filho**
}

\begin{abstract}
HARDY, E. E. et al. A prätica da amamentação no município de Paulínia, Estadı de São Paulo, Brasil. Rev. Saúde públ., S. Paulo, 16:337-45, 1982.

RESUMO: Em 1977 foi realizado um inquérito no município de Paulínia, Estado de São Paulo (Brasil), com a finalidade de se estudar o aleitamento materno das crianças de alé dois anos de idade. $O$ inquérito forneceria dados a serem utilizados para avaliar lim programa de estímulo ao aleitamento materno, a ser desenvolvido mais tarde. A idade média das crianças no desmame foi 3.3 meses, e 2.2 meses ao começar a tomar leite em mamadeira. No final do primeiro mês, $74 \%$ das crianças estavam sendo amamentadas e $\mathbf{5 7 \%}$ estavam com aleitamento exclusivo. As crianças de mães com 28 anos ou mais tiveram uma amamentação exclusiva significativamente mais longa que as crianças de mães mais jovens. A residência, urbano/rural, e o sexo das crianças não influíram na duração da amamentação.
\end{abstract} Brasil.

UNITERMOS: Aleitamento materno. Inquéritos nutricionais, Paulínia, SP,

\section{INTRODUÇAO}

Nos últimos anos tem-se observado no Brasil um grande interesse pelo aleitamento materno, tanto de parte das autoridades, como dos profissionais na área da saúde. $O$ interesse resulta de evidências que mostram as vantagens do leite materno para a saúde da criança ${ }^{2,9}$ e dos resultados de diversas pesquisas que apontam um desmame cada vez mais precoce ${ }^{8,12}$, incluindo uma proporção de até 10 a $15 \%$ de mulheres que não amamentam seus filhos 13,17. Essas observações fizeram com que diversos autores assinalassem a necessidade de desenvolver ações que levassem um número maior de mulheres a amamentar, e a um desmame mais tardio ${ }^{14,15}$. Com este objetivo, foi planejado um programa de estímulo ao aleitamento materno ${ }^{5,7}$, a ser desenvolvido no município de Paulínia, no Estado de São Paulo. Considerando a pouca experiência existente sobre o efeito das ações de estímulo e a necessidade de se utilizar os recursos disponiveis da maneira mais eficaz possivel, optou-se por começar desenvol-

\footnotetext{
* Do Departamento de Tocoginecologia da Faculdade de Ciências Médicas da UNICAMP. Caixa Postal 1452 - 13100 - Campinas, SP - Brasil.

* Do Centro de Pesquisas Materno Infantis de Campinas (CEMICAMP) - 13100 - Campinas, SP - Brasil.

**: Do Departamento de Pediatria da Faculdade dt Ciências Médicas da UNICAMP.
} 
HARDY, E.E. et al. A prática da amamentação no município de Paulínia, Estado de São Paulo, Brasil. Rev. Saúde públ., S. Pa:ilo, 16:337-45, 1982.

vendo um inquérito para obter informações de base, a serem utilizadas para posterior avaliação do programa.

Este trabalho apresenta os resultados do inquérito, desenvolvido em meados de 1977, que visou coletar informações sobre a amamentação de todas as crianças com até dois anos de idade.

\section{MATERIAL E METODO}

\section{Sujeitos estudados}

Foram incluidas no estudo todas as crianças vivas que haviam completado no máximo dois anos de idade, no dia em que. foi feita a entrevista correspondente, bem como as crianças falecidas que estarjam no máximo com dois anos na data da entrevista.

Os nomes e endereços das crianças menores de dois anos, que moravam no município, foram obtidos de diversas fontes. A mais importante foi o Censo, realizado em fins de 1976, pela Divisão de Planejamento e Coordenação da Prefeitura. Todos os formulários preenchidos na ocasião foram revisados. O Cartório do municipio forneceu os endereços das crianças registradas entre novembro de 1976 e junho de 1977.

No Ambulatório de Pré-Natal do Centro de Saúde Escola de Paulinia, obtiveram-se os nomes e endereços das pacientes que dariam à luz entre novembro de 1976 e junho de 1977; e obteve-se uma lista das crianças na faixa etária procurada, que eram controladas pelo Ambulatório de Puericultura. Finalmente, obteve-se uma lista das mulheres de Paulinia que haviam tido o parto na Maternidade da Santa Casa de Campinas nos dois anos anteriores ao inquérito.

Todos os dados fornecidos pelos Ambulatórios de Paulínia e pela Maternidade foram confrontados com os do Censo e do Cartório, para completar a lista das crianças vivas ou falecidas com idade de zero a dois anos.

\section{Metodologia}

Para fazer as entrevistas foram utilizados dois questionários estruturados, previamente testados. O primeiro permitia saber se a criança procurada morava no endereço. havia se mudado ou falecido. Esse questionário também permitia anotar o novo endereço e identificar a mãe da criança. No segundo questionário, era conferida a idade da criança e registrada a informação relativa à amamentação. Este questionário permitia saber com que idade estava a criança quando havia começado a tomar leite em mamadeira e quando havia sido desmamada.

Os entrevistadores, de ambos os sexos. tram estudantes (bolsistas da Prefeitura) selecionados após treinamento teórico-prático de 10 horas. Como os entrevistadores tinham aulas e alguns também trabalhavam. quase todas as atividades do inquérito foram desenvolvidas durante os fins de semana dos meses de junho e julho de 1977.

Com a finalidade de unificar critérios. foram utilizados manuais com instruções para o desenvolvimento das entrevistas, para a revisão dos questionários completados e para a codificaçăo.

Em torno de 5\% dos endereços designados a cada entrevistador foram visitados por uma supervisora, com o objetivo de verificar se havia sido feita a entrevista e conferir alguns dados.

\section{Análise dos dados}

Definiu-se como aleitamento exclusivo a situação em que a criança recebeu só leite materno, e como aleitamento misto a amamentação complementada com outro leite, fornecido em mamadeira. A duração do aleitamento inclui o tempo total em que a criança esteve com aleitamento materno; exclusivo e/ou misto.

A duração do aleitamento e do aleitamento exclusivo foi estudada pelo método da tabela de vida ${ }^{10}$, que permite analisar a proporção de crianças que em cada mês de vida abandonaram totalmente - aleitamento ou o aleitamento exclusivo (iniciaram mamadeira). O método também permite estudar a proporção de crianças que 
HARDY. E.E. et al. A prática da amamentação no municipio de Paulínia, Estado de São Paulo, Brasil. Rev. Saúde públ., S. Paulo, 16:337-45, 1982.

continuaram sendo amamentadas no fim de cada mês (taxa de continuação).

O método da tabela de vida é utilizado fundamentalmente nos estudos de mortalidade. Pretende estimar a probabilidade que tem uma coorte de indivíduos de morrer (ou sobreviver) em cada ano (ou mês) de vida, sempre que sua mortalidade fosse igual à observada nos individuos que no momento do estudo tinham de um até $n$ anos (ou meses) de idade.

Aplicada à duração do aleitamentu, o método de tabela de vida pretende estimar a probabilidade de interromper (ou continuar) a amamentação de uma coorte de mães que tiveram a mesma experiência de amamentação que as mães estudadas, que interromperam o aleitamento em diferentes jdades dos filhos. Nesse caso, cada interrupção tota! do aleitamento ou cada início de mamadeira equivale a uma morte no método original que estuda mortalidade.

O método da tabela de vida tem o mérito de calcular o risco mês a mês, incluindo no denominador só os casos que estão expostos ao risco em estudo (morrer, iniciar mamadeira, interromper aleitamento). Daí parecer um método apropriado para medir a duração do aleitamento e para comparar a experiência de distintas coortes de mães.

$\mathrm{O} \chi^{2}$ utilizado ${ }^{16}$ compara todos os valores das taxas mensais de continuaçāo para os grupos que se estudam, o que equivale à comparação das curvas que resultariam das taxas. (Tabelas 3 e 4 ).

Para a tabela de vida, as crianças nunca amamentadas pela mãe foram consideradas como desmamadas no primeiro mês de vida.

\section{RESULTADOS}

Foram identificadas 797 crianças que teriam nascido até dois anos antes da data da entrevista. Foram encontradas 642 $(81 \%)$, sendo que 32 tinham mais de dois anos no dia da entrevista. As famílias de 8 das crianças estavam viajando, de 81 haviam se mudado e de 66 eram desconhecidas no endereço ou este não foi achado. Os resultados apresentados foram obtidos ao entrevistar 555 mães e uma avó, que forneceram informações sobre 610 crianças.

$\mathrm{Na}$ entrevista, a idade média das mães foi 26,5 anos. A grande maioria $(82,4 \%)$ tinha um só filho de até dois anos de idade e mais da metade $(67,3 \%)$ morara na área urbana de Paulínia. Pouco mais da metade. das crianças era do sexo feminino (Tabela 1).

TA B E L A 1

Distrib ição percentual das $\mathbf{5 5 5}$ mães segundo idade na entrevista, lugar de residència, númelo e sexo dos filhos de até dois anos de idade, no município de Paulínia, SP, 1977.

\begin{tabular}{cc} 
Idade na entrevista & $\%$ \\
$\mathbf{1 5}-\mathbf{1 9}$ & $\mathbf{9 , 4}$ \\
$\mathbf{2 0 - 2 4}$ & 32,4 \\
$\mathbf{2 5 - 2 9}$ & 29.7 \\
$30-34$ & 18,5 \\
$\mathbf{3 5 - 4 5}$ & 10,0 \\
& \\
Lugar de residência & $\%$ \\
Urbano & 67,3 \\
Rural & 32,7 \\
Número de fjlhos * & $\%$ \\
1 & $\mathbf{9 0 , 4}$ \\
$\mathbf{2}$ & $\mathbf{9 , 5}$ \\
Sexo dos filhos & $\%$ \\
Feminino & $\mathbf{5 3 , 0}$ \\
Masculino & $\mathbf{4 7 . 0}$ \\
\hline
\end{tabular}

* Uma mãe tinha 3 filhos de até dois anos.

$\mathrm{Na}$ entrevista, a idade média das crianças foi 11,9 meses. Estavam sendo amamentadas $148(24,3 \%)$; já haviam sido desmamadas $388(63,6 \%)$ e nunca haviam sido amamentadas $74(12,1 \%)$, incluindo 10 das 11 que haviam falecido.

A idade média ao começar a tomar leite em mamadeira foi de 1.8 meses nas 459 crianças que usavam mamadeiras e de 2.2 meses nas 385 que não haviam sido álimentadas com mamadeira a partir do nascimento. 
HARDY, E.E. et al. A prática da amamentação no município de Paulínia, Estado de São Paulo, Brasil. Rev. Saúde públ., S. Paulo, 16:337-45, 1982.

As taxas de continuação do aleitamento e do aleitamento exclusivo são apresentadas na Tabela 2. No final do primeiro mês de vida, $74 \%$ das crianças estavam sendo amamentadas e $57 \%$ estavam com aleitamento exclusivo. No sexto mês estas percentagens caíram para $34 \%$ e $18 \%$, respectivamente. No final do primeiro ano, um quinto $(22 \%)$ das crianças estava sendo amamentada, sendo que $12 \%$ ainda não recebiam outro leite em mamadeira.

T A B E L A 2

Taxa de continuação e desvio padrão (DP) do aleitamento e do aleitamento exclusivo durante o primeiro ano de vida de crianças, no município de Paulinia, SP, 1977.

\begin{tabular}{|c|c|c|c|c|}
\hline \multirow{2}{*}{$\begin{array}{l}\text { Idade } \\
\text { em meses }\end{array}$} & \multicolumn{2}{|c|}{ Aleitamento } & \multirow{2}{*}{$\begin{array}{c}\text { Aleitamento } \\
\text { Taxa }\end{array}$} & \multirow{2}{*}{$\begin{array}{l}\text { exclusive } \\
\mathrm{DP}\end{array}$} \\
\hline & Taxa & $\mathrm{DP}$ & & \\
\hline 1 & 0.74 & 0,02 & 0,57 & 0.02 \\
\hline 2 & 0.62 & 0,02 & 0.43 & 0,02 \\
\hline 3 & 0,49 & 0.02 & 0,31 & 0.02 \\
\hline 4 & 0,41 & 0,02 & 0,26 & 0,02 \\
\hline 5 & 0,35 & 0,02 & 0,21 & 0,02 \\
\hline 6 & 0.34 & 0,02 & 0,18 & 0,02 \\
\hline 7 & 0,30 & 0,02 & 0,15 & 0,02 \\
\hline 8 & 0,27 & 0,02 & 0,14 & 0,02 \\
\hline 9 & 0,24 & 0,02 & 0,12 & 0,01 \\
\hline 10 & 0,24 & 0,02 & 0,12 & 0,01 \\
\hline 11 & 0.22 & 0,02 & 0,12 & 0.01 \\
\hline 12 & 0.22 & 0,02 & 0.12 & 0,01 \\
\hline
\end{tabular}

A idade média no desmame foi de 3.3 meses, sendo que a mediana do aleitamento foi observada no terceiro mês e a do aleitamento exclusivo no segundo mês (Tabela 3).

A duração do aleitamento e do aleitamento exclusivo em diversos sub-grupos é apresentada nas Tabelas 3 e 4 . As taxas de continuação foram calculadas somente até o oitavo mês, porque o número de casos nos meses posteriores não permitiu aplicar a tabela de vida.

Não se observou diferença significativa na duração do aleitamento, ao comparar as crianças de mães de até 27 anos com as de mães com 28 anos ou mais, nem ao comparar as que moravam na zona urbana com as da área rural. Também não houve diferenças na duração do aleitamento ao comparar meninos e meninas (Tabela 3).

A duração do aleitamento exclusivo estudada pela tabela de vida foi significativamente maior nas crianças de mães com 28 anos ou mais, comparadas com os filhos de mulheres mais jovens. No primeiro mês, $60 \%$ das crianças de mães com 28 anos ou mais estavam com aleitamento exclusivo comparadas com $46 \%$ das crianças de mães com até 27 anos. Aos seis meses as percentagens haviam diminuido para $22 \%$ e $13 \%$. respectivamente (Tabela 4 ).

A duração do aleitamento exclusivo não foi significativamente diferente nas meninas comparadas com os meninos, nem nas que moravam na área urbana comparadas com as da área rural (Tabela 4).

\section{DIsCUSSAO E CONCLUSÃo}

As publicações brasileiras referentes à duração do aleitamento materno são pouco numerosas. Em geral, referem-se a pacientes de hospitais ou de ambulatórios, razão pela qual os resultados não podem ser considerados representativos da população. Neste trabalho, os dados apresentados correspondem à maior parte das crianças de até dois anos de idade, que moravam no municipio de Paulinia por ocasiāo do inquérito.

A dificuldade que tivemos para encontrar as familias das crianças identificadas poderia atribuir-se, principalmente, a duas causas: o fato da população de Paulínia ser muito móvel e que muitos dos endereços das familias eram sitios, chácaras ou beira de estradas. Pelo menos $52 \%$ das famílias não encontradas havia mudado, e de 66 casos em que o endereço não foi achado ou a família procurada era desconhecida, 54 corresponderam a endereços na área rural.

$\mathrm{Na}$ medida em que as crianças não encontradas tivessem uma amamentação significativamente diferente da observada no grupo estudado, poderia haver um viés nos 
HARDY, E.E. et al. A prática da amamentação no município de Paulínia, Estado de São Paulo, Brasil. Rev. Saúde públ., S. Paulo, 16:337-45, 1982.

T A B E L A 3

Taxa de continuação e desvio padrão (DP) do aleitamento de crianças e respectiva diferença, segundo idade da mãe na entrevista, sexo da criança c l'gar de residência, no município de Paulínia, SP, 1977.

\begin{tabular}{|c|c|c|c|c|c|}
\hline \multicolumn{6}{|c|}{ Idade da mãe } \\
\hline \multirow{2}{*}{$\begin{array}{l}\text { Idade } \\
\text { cm meses }\end{array}$} & \multicolumn{2}{|c|}{ Até 27 anos } & \multirow{2}{*}{$\begin{array}{c}28 \text { anos } \\
\text { Taxa }\end{array}$} & \multirow{2}{*}{$\begin{array}{l}\text { mais } \\
\mathrm{DP}\end{array}$} & \multirow[t]{2}{*}{ Diferença } \\
\hline & Taxa & $\mathrm{DP}$ & & & \\
\hline 1 & 0,73 & 0,02 & 0,76 & 0,03 & $-0,03$ \\
\hline 2 & 0,60 & 0,03 & 0,65 & 0,03 & $-0,05$ \\
\hline 3 & 0,46 & 0,03 & 0,55 & 0,03 & $-0,09$ \\
\hline 4 & 0,38 & 0,03 & 0,47 & 0,03 & $-0,09$ \\
\hline 5 & 0,31 & 0,03 & 0,43 & 0,03 & $-0,12$ \\
\hline 6 & 0,29 & 0,02 & 0,39 & 0,03 & $-0,10$ \\
\hline 7 & 0,25 & 0,02 & 0,35 & 0,03 & $-0,10$ \\
\hline 8 & 0,23 & 0,02 & 0.31 & 0.03 & -0.08 \\
\hline \multicolumn{6}{|c|}{ Sexo da criança } \\
\hline Idade & \multicolumn{2}{|c|}{ Feminino } & \multicolumn{2}{|c|}{ Masculino } & Diferença \\
\hline $\mathrm{cm}$ meses & Taxa & DP & Taxa & $\mathrm{DP}$ & \\
\hline 1 & 0,73 & 0,03 & 0,75 & 0,03 & $-0,02$ \\
\hline 2 & 0,61 & 0,03 & 0,63 & 0,03 & $-0,02$ \\
\hline 8 & 0,48 & 0,03 & 0,50 & 0,03 & -0.02 \\
\hline 4 & 0,41 & 0,03 & 0.42 & 0,03 & -0.02 \\
\hline 5 & 0,34 & 0,03 & 0,37 & 0,03 & -0.03 \\
\hline 6 & 0,31 & 0,03 & 0,35 & 0,03 & $-0,04$ \\
\hline 7 & 0,27 & 0,03 & 0,30 & 0,03 & $-0,03$ \\
\hline 8 & 0,26 & 0.03 & 0.26 & 0.03 & - \\
\hline \multirow{3}{*}{$\begin{array}{c}\text { Idade } \\
\text { cm meses }\end{array}$} & \multicolumn{4}{|c|}{ Lugar de residência } & \\
\hline & \multicolumn{2}{|c|}{ Urbano } & \multicolumn{2}{|c|}{ Rural } & Diferença \\
\hline & Taxa & $\mathrm{DP}$ & Taxa & DP & \\
\hline 1 & 0,74 & 0,02 & 0,73 & 0,03 & $+0,01$ \\
\hline 2 & 0,61 & 0,03 & 0,65 & 0,03 & $-0,04$ \\
\hline 3 & 0,48 & 0,03 & 0,51 & 0,03 & $-0,03$ \\
\hline 4 & 0,40 & 0,03 & 0,45 & 0,03 & $-0,04$ \\
\hline 5 & 0,33 & 0,02 & 0,40 & 0.03 & $-0,08$ \\
\hline 6 & 0,30 & 0,02 & 0,39 & 0.03 & $-0,09$ \\
\hline 7 & 0,26 & 0,02 & 0,34 & 0,03 & $-0,08$ \\
\hline 8 & 0,22 & 0,02 & 0.32 & 0.03 & $-0,10$ \\
\hline
\end{tabular}


HARDY, E.E. et al. A prática da amamentação no município de Paulínia, Estado de São Paulo, Brasil. Rev. Saúde públ., S. Paulo, 16:337-45, 1982.

T A B E L A 4

Taxa de continuação e desvio padrão (DP) do aleitamento exclusivo de crianças e respectiva diferença, segunåo idade da mãe na entrevista, sexo da criança e lugar de residência, no município de Paulínia, SP, 1977

\begin{tabular}{|c|c|c|c|c|c|}
\hline \multicolumn{6}{|c|}{ Idade da mãe } \\
\hline \multirow{2}{*}{$\begin{array}{c}\text { Idade } \\
\mathrm{cm} \text { meses }\end{array}$} & \multicolumn{2}{|c|}{ Até 27 anos } & \multirow{2}{*}{$\begin{array}{c}28 \text { anos } \\
\text { Taxa }\end{array}$} & \multirow{2}{*}{$\begin{array}{l}\text { mais } \\
D P\end{array}$} & \multirow[t]{2}{*}{ Diferença } \\
\hline & Taxa & $\mathrm{DP}$ & & & \\
\hline 1 & 0,46 & 0,03 & 0,60 & 0,03 & $-0,14$ \\
\hline 2 & 0,33 & 0.03 & 0,46 & 0,03 & $-0,13$ \\
\hline 3 & 0,23 & 0.02 & 0,35 & 0,03 & -0.12 \\
\hline 4 & 0.19 & 0,02 & 0,29 & 0,03 & $-0,10$ \\
\hline 5 & 0,15 & 0,02 & 0,25 & 0,03 & $-0,10$ \\
\hline 6 & 0,13 & 0,02 & 0,22 & 0,03 & $-0,09$ \\
\hline 7 & 0,10 & 0,02 & 0,19 & 0,03 & $-0,09$ \\
\hline 8 & 0,09 & 0,02 & 0,17 & 0,03 & -0.08 \\
\hline \multicolumn{6}{|c|}{ Sexo da criança } \\
\hline Idade & \multicolumn{2}{|c|}{ Feminino } & \multicolumn{2}{|c|}{ Masculino } & \multirow[t]{2}{*}{ Diferença } \\
\hline em meses & Taxa & $\mathrm{DP}$ & Taxa & $\mathrm{DP}$ & \\
\hline 1 & 0,58 & 0,03 & 0,57 & 0,03 & $+0,01$ \\
\hline 2 & 0,42 & 0,03 & 0,42 & 0,03 & - \\
\hline 3 & 0,31 & 0,03 & 0,29 & 0,03 & $\lceil 0,02$ \\
\hline 4 & 0,26 & 0,03 & 0,25 & 0,03 & $+0,01$ \\
\hline 5 & 0,20 & 0,02 & 0.21 & 0,03 & -0.01 \\
\hline 6 & 0.17 & 0,02 & 0,20 & 0,03 & $-0,03$ \\
\hline 7 & 0.14 & 0,02 & 0.17 & 0,03 & $-0,03$ \\
\hline 8 & 0,13 & 0,02 & 0,16 & 0,03 & $-0,03$ \\
\hline \multicolumn{6}{|c|}{ Lugar de residência } \\
\hline Idade & \multicolumn{2}{|c|}{ Urbano } & \multicolumn{2}{|c|}{ Rural } & Diferença \\
\hline em meses & Taxa & DP & Taxa & $\mathrm{DP}$ & \\
\hline 1 & 0,58 & 0,03 & 0,57 & 0,03 & $+0,02$ \\
\hline 2 & 0,43 & 0,03 & 0,42 & 0,03 & +0.01 \\
\hline 3 & 0.32 & 0,02 & 0,29 & 0,03 & $+0,03$ \\
\hline 4 & 0,26 & 0,02 & 0,25 & 0,03 & $+0,02$ \\
\hline 5 & 0.20 & 0,02 & 0,21 & 0,03 & $-0,01$ \\
\hline 6 & 0.17 & 0,02 & 0,20 & 0,03 & $-0,03$ \\
\hline 7 & 0,14 & 0.02 & 0,17 & 0,03 & $-0,04$ \\
\hline 8 & 0,12 & 0,02 & 0,16 & 0,03 & -0.04 \\
\hline
\end{tabular}

$\chi^{2}$ significativo $(\mathrm{p}<0,001)$ 
FARDY, E.E. et al. A prática da amamentação no município de Paulínia, Estado de São Paulo, Brasil. Rev. Saúde públ., S. Paulo, 16:337-45， 1982.

nossos resultados. Ao comparar o grupo da área urbana com o grupo da área rural, não observamos diferenças significativas na duração do aleitamento $11 \mathrm{em}$ do aleitamento exclusivo. Esta falta de diferença poderia ser real, ou dever-se a: falta de informasão dos casos perdidos - que poderiam ter sido mais genuinamente rurais -; ou ao tamanho reduzido do municipio, que implica uma divisão urbano-rural relativamente artificial: ou a proximidade de uma cidade como Campinas, cuja influência poderia abranger toda a população de Paulínia.

A idade média ao começar a receber leite tm mamadeira foi calculada a partir das crianças que usavam mamadeira, excluindo-se 7 casos que passaram diretamente a tomar leite do copo ou caneca. A idade média no desmame foi calculada com base nas crianças desmamadas totalmente por ocasião da entrevista. Os valores observados poderiam ser diferentes se todas as crianças tivessem sido consideradas.

O fato das crianças de sexo masculino terem um valor maior para a familia em algumas culturas (o que poderia refletir-se t'm uma amamentação mais prolongada dos meninos) nos levou a estudar a relação entre sexo das criançás e duração do aleitamento e do aleitamento exclusivo. Não houve difelença significativa entre meninos e meninas, resultado que coincide com a falta de associação observada en um grupo de puérperas s, bem como em uma amostra da zona urbana de Teresina, no Piauí ${ }^{15}$.

É difícil comparar os resultados de nosso trabalho com os obtidos por outros autores no Brasil, porque as populações estudadas são diferentes. Levando em consideração esta diferença metodológica, serão discutidas algumas características do aleitamento materno.

Na medida que o desmame total vai ocorrendo cada vez mais precocemente, chega un momento em que um certo número de crianças nunca é amamentada. Em Paulinia, $12,3 \%$ das crianças nunca havia sido amamentada, sendo que das 388 desmamadas, por ocasião da entrevista, $48 \%$ já havia sido totalmente desmamadas no segundo mês de vida. Em Londrina, $12,8 \%$ das crianças não foi amamentada e $31 \%$ havia sido desmamada no primeiro mês de vida ${ }^{13}$. Em Teresina a mediana da duração do aleitamento foi de 3 meses e $13,3 \%$ das crianças nunca foi amamentada $1 \pm$.

Quanto mais cedo a criança começa a tomar outro leite, mais precoce parece ser 0 desmame ${ }^{*}$. Em Paulinia, $43 \%$ das crianças iniciou o uso de mamadeira durante 0 primeiro mês de vida e $26 \%$ havia sido desmamada nesse mesmo periodo. A grande maioria das crianças foi desmamada no mesmo mês em que iniciou uso de mamadeira. A situaçāo foi semelhante no estudo de pacientes de Ambulatório em Londrina 11 já que, no primeiro mês de vida, $43,6 \%$ das crianças começou com aleitamento artificial e $42,1 \%$ havia sido desmamada.

A duração do aleitamento exclusivo em Paulinia foi significativamente maior nas crianças com mães de 28 anos ou mais de idade, comparadas com aquelas cujas mães tinham até 27 anos. A associação entre idade da mãe e duração da amamentação havia sido observada em 1975, em um grupo de mulheres residentes no Distrito de São Paulo, onde a maior percentagem $(16,2 \%)$ das crianças amamentadas por menos de três semanas correspondeu a mães de 15-24 anos, comparada com $6,9 \%$ no grupo com 35 anos ou mais ${ }^{17}$.

Um inquérito importante para estudar a incidência e duração da amamentação nas regiōes metropolitanas de São Paulo e Recife foi desenvolvido pelo CEBRAP em $1981^{1}$. Os autores estudaram as caracteristicas da amamentação das crianças no momento da entrevista, portanto não obtiveram informaçōes sobre a idade em que cada criança foi desmamada. Ao mesmo tempo não utilizaram a tabela de vida para a análisc. Apesar desta điferença metodológica, é possivel fazer algumas comparaçóes. $O$ estudo do CEBRAP encontrou $9 \%$ de māes que nunca amamentou em São Paulo e $12 \%$ em Recife $(12,3 \%$ em Paulínia). 
HARDY, E.E. et al. A prática da amamentação no município de Paulínia, Estado de São Paulo, Brasil. Rev. Saúde públ., S. Paulo, 16:337-45, 1982.

O tempo médio de aleítamento foi estimado em 89 dias em São Paulo e 66 dias em Recife, o que se compara com a taxa de continuação de $49 \%$ ao fim do terceiro mês de vida em Paulínia, que permite estimar um tempo médio de aleitamento apenas inferior a 90 dias.

Salvo as diferenças metodológicas, os resultados parecem indicar que a conduta das mães do município de Paulínia, em 1977, era semelhante a observada em São Paulo, em 1981. Considerando-se que a duração do aleitamento está fortemente influenciada pelo atendimento obstétrico ${ }^{3}$ e pediátrico ${ }^{5}$; que as mães de Paulinia têm o seu parto em Hospitais (principalmente de Campinas), e que as condutas médicas são similares na grande São Paulo e na sua área de influência, não é estranho que os resultados sejam parecidos. Esta semelhança poderia refletir, também, a difusão de símbolos culturais, como é o uso da mamadeira, desde a grande cidade até municipios semi-rurais, a $100 \mathrm{~km}$ de distância.

Independentemente da possivel explicação do resultado observado, não há dúvida de que o desmame precoce e a alta proporçāo de mulheres que não amamentam não são fenômenos reştritos às grandes cidades, mas encontrados Bem além delas. A causa, entre outras, poderia ser a influência dos meios de comunicação, as normas de conduta médica ou uma combinação de ambas.

HARDY, E. E. et al. [Breastfeeding in the town of Paulinia, S. Paulo State (Brazil)]. Rev. Saúde públ., s. Paulo, 16:337-45, 1982.

ABSTRACT: In 1977 a survey $u$ as carried out in the municipality of Paulinia, State of $\mathrm{S}$. Paulo, with the purpose of obtaining information on the breastfeeding of children up to two years of age. The survey would provide base line data for the evaluation of a breastfeeding promotion pro. gram, that was to be carried out later. The children's mean age at weaning was 3.3 months, and 2.2 months when they started bottle feeding. At the end of the first month, $74 \%$ of the children were breastfed and $57 \%$ were fully breastfed. Children whose mothers were at least 28 years old were fully breastfed for a significantly longer period than children with younger mothers. Urban/rural residence and the sex of the children had no influence on the duration of breastfeeding.

UNITERMS: Breastfeeding. Nutritional surveys, Paulinia, SP, Brazil.

\section{REFERENCIAS BIBLIOGRAFICAS}

1. BERQUO, E.; SPINDEL, S.: SINGER, P.; REA, M. \& CUKIER, R. Estudo do aleitamento materno na Grande São Paulo $e$ a Grande Recife. São Paulo, CEBRAP, 1981.

2. CUNNINGHAM; A.S. Morbidity in breast fed and artificially fed infants. $J, P e$ diat. 90:726-9, 1977.

3. FAUUNDES, A. O médico e o estimulo da lactação. [Apresentado no Seminário sobre Estímulo ao Aleitamento Natural, Campinas, 1977$]$.
4. GREINER, T.H. Infant food advertising and malnutrition in St. Vincent. Ithaca, NY, 1977. [Master - Cornell University].

5. HALPERN, S.R.; SELLARS, W.A.; JOHNSON, R.B.; ANDERSON, D.W.; SAPERSTEIN, S. \& SHANNON, S. Factors influencing breastfeeding. South Med. J., 65:100-2, 1972.

6. HARDY, E.E. Alguns fatores relacionados com o aleitamento materno e descrição de dois programas de estímulo. [Apre- 
HARDY, E.E. et al. A prática da amamentação no municipio de Paulínia, Estado de São Paulo, Brasil. Rev. Saúde puibl., S. Paulo, 16:337-45, 1982.

sentado ao I Seminário Regional sobre Estímulo ao Aleitamento Materno. Região Nordeste, Recife, 1979].

7. HARDY, E.E.: VICHI, A.M.; BAFAMONDES, M.Y.M. de: PINOTTI, J.A. \& MARTINS FILHO, J. Programa comunitário de estímulo do aleitamento materno: descriçăo de uma experiência. Rev. Ass. med. bras., 27:288-90, 1981.

3. MARTINS FILHO, J. Contribuiçăo ao estudo do aleitamento materno em Campinas. Campinas, 1976. [Tese de Livre Docência - Faculdade de Clências Médicas UNICAMP].

9. PLANK, S.J. \& MILANESI, M.L. Infant feeding and infant mortality in rural Chile. Bull. WHO, 48:293-310, 1973.

10. TIETZE, C. Intra-uterine contraception: recommended procedures for data analysis. Stud. Fam. Plann., (Suppl). Apr. 1967.

11. THOMSON, Z. Estudo da prática do aleitamento materno. Londrina, 1975. [Tese de Doutoramento - Centro de Ciências da Saúde da Universidade Estadual de Londrina].
12. THOMSON, Z. Estudo da prática do aleitamento materno em um grupo populacional, Londrina, PR. J. Pediat., Rio de Janeiro, 45:379-85, 1978.

13. THOMSON, Z. Fatores associados ao desmame em um grupo populacional, Londrina, PR. J. Pediat., Rio de Janeiro, 46:93-8, 1979.

14. VALQUIST, B.; STAPLETON, T. \& BÉHAR, M. New urban families. Acta paediatr. Acad. Sci. Hung., 13:95-9, 1972.

15. VIEIRA, J,N. Estudo sobre o aleitamento materno na zona urbana de Teresina. Recife, 1979. [Dissertação de Mestrado Universidade Federal de Pernambuco].

16. WATSON, F.R. Statistical methods in cancer research. In: Donegan, W.L. \& Spratt, J.S., eds. Cancer of the breast. Philadelphia, Saunders, 1979. p. 613-63.

17. YUNES, J. \& RONCHEZEL, V.S.C. Estudo da lactação em mulheres do distrito de São Paulo, Brasil. Rev. Saúde pübl., S. Paulo, 9:191-213, 1975.

Recebido para publicasão em 15/07/1982 Aprovado para publicạ̧̃o em 20/09/1982 